\title{
A critical exploration of the International Classification of Functioning, Disability, and Health (ICF) framework from the perspective of oncology: recommendations for revision
}

\author{
This article was published in the following Dove Press journal: \\ Journal of Multidisciplinary Healthcare \\ 7 March 2013 \\ Number of times this article has been viewed
}

\author{
Catherine C Bornbaum' \\ Philip C Doyle ${ }^{1-3}$ \\ Elizabeth Skarakis-Doyle ${ }^{1,2}$ \\ Julie A Theurer ${ }^{2,3}$ \\ 'Graduate Program in Health and \\ Rehabilitation Sciences, ${ }^{2}$ School \\ of Communication Sciences \\ and Disorders, ${ }^{3}$ Department \\ of Otolaryngology - Head and Neck \\ Surgery, Schulich School of Medicine \\ and Dentistry, The University \\ of Western Ontario, London, \\ ON, Canada
}

\begin{abstract}
Background: In 2001, the World Health Organization developed the International Classification of Functioning, Disability, and Health (ICF) framework in an effort to attend to the multidimensional health-related concerns of individuals. Historically, although the ICF has frequently been used in a rehabilitation-based context, the World Health Organization has positioned it as a universal framework of health and its related states. Consequently, the ICF has been utilized for a diverse array of purposes in the field of oncology, including: evaluating functioning in individuals with cancer, guiding assessment in oncology rehabilitation, assessing the comprehensiveness of outcome measures utilized in oncology research, assisting in health-related quality of life instrument selection, and comparing the primary concerns of health professionals with those of their patients.
\end{abstract}

Discussion: Examination of the ICF through the lens of cancer care highlights the fact that this framework can be a valuable tool to facilitate comprehensive care in oncology, but it currently possesses some areas of limitation that require conceptual revision; to this end, several recommendations have been proposed. Specifically, these proposed recommendations center on the following three areas of the ICF framework: (1) the replacement of the term "health condition" with the more inclusive and dynamic term "health state;" (2) the continuing development and refinement of the personal factors component to ensure issues such as comorbidities can be accounted for appropriately; and (3) the inclusion of a mechanism to account for the subjective dimension of health and functioning (eg, quality of life).

Summary: It is through the expansion of these conceptual parameters that the ICF may become more relevant and applicable to the field of oncology. With these important revisions, the ICF has the potential to provide a broader biopsychosocial perspective of care that captures the diverse range of concerns that arise throughout the continuum of care in oncology.

Keywords: ICF, International Classification of Functioning, Disability, and Health, cancer, quality of life, personal factors, health condition

\section{Background}

Cancer is a disease of multiple types, sites, and causes. Statistics indicate that it is the leading cause of death in economically developed countries and the second leading cause of death in developing nations. ${ }^{1}$ Recent data suggest that this prevalence translated to approximately 12.7 million diagnoses of cancer and 7.6 million cancerrelated deaths in 2008. ${ }^{2}$ Importantly, concerns related to cancer extend beyond the pervasiveness of the disease to also include the myriad of consequences that stem from it. Due to the current forms of treatment available (eg, chemotherapy, radiotherapy,
Correspondence: Catherine C Bornbaum Graduate Program in Health and Rehabilitation Sciences, The University of Western Ontario (UWO), Room 2200, Elborn College, London, ON N6G IHI, Canada

Tel +15196612111

$\mathrm{Fax}+\mathrm{I} 5198502469$

Email cbornba@gmail.com 
surgery), there are often significant consequences related to the functioning and quality of life (QOL) of individuals with cancer. ${ }^{3}$ Additionally, owing to improved surveillance and treatment methods, survival rates of cancer have improved over time, ${ }^{4}$ creating the need to recognize and attend to a variety of concerns unique to cancer survivorship. ${ }^{5}$ In order to identify and attend to the numerous potential concerns of individuals diagnosed with cancer, it may be useful to utilize an overarching framework to guide the provision of care.

Historically, medicine was delivered according to a biomedical approach to care that emphasized the physical aspects of disease. However, current frameworks of care have expanded beyond this biomedical focus. In an effort to identify and attend to the multidimensional health-related concerns of individuals, in 2001 the World Health Organization (WHO) developed the International Classification of Functioning, Disability, and Health (ICF; Figure 1). ${ }^{6}$ The ICF framework was designed to provide a common basis for terminology, as well as understanding and classifying health and its related states, determinants, and outcomes. The ICF consists of a unified language for describing these factors in order to enhance communication among practitioners, researchers, policy makers, and the general public. In terms of its theoretical position, the ICF is guided by the biopsychosocial model. ${ }^{7}$ This model emphasizes the multidimensional nature of health concerns and demonstrates functional interdependence between one's physical, psychological, and social functioning. Owing to its roots in the interactionist biopsychosocial model, the ICF was intended to be universally applicable to any individual in any state of health or infirmity. Additionally, with respect to terminology, the ICF was designed to be as neutral

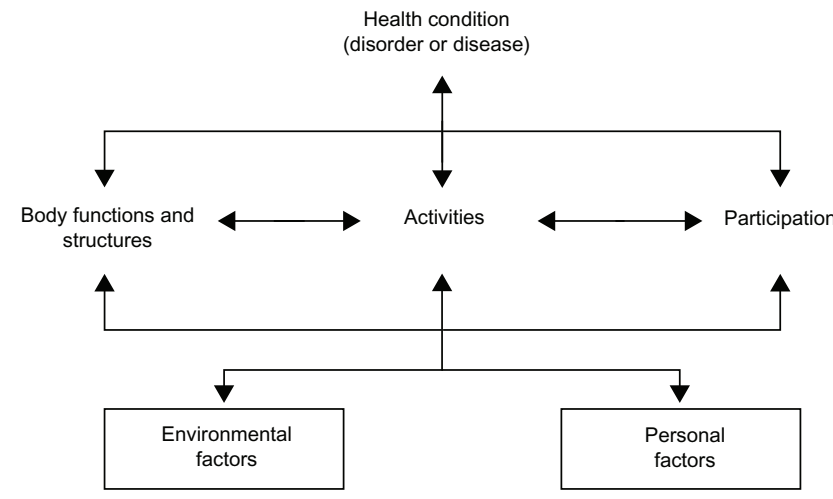

Figure I The International Classification of Functioning, Disability, and Health. Reproduced with permission of the World Health Organization (Permission ID: 108752). Those interested in learning more about the International Classification of Functioning, Disability, and Health are encouraged to review the beginner's guide developed by the World Health Organization (available from: http://www.who.int/ classifications/icf/training/icfbeginnersguide.pdf). as possible so as to avoid any "undue negative connotation." In essence, the underlying principles of the ICF suggest that it aims to serve as a neutral, comprehensive, and universally applicable framework of health and functioning. It would, therefore, appear that the ICF has the potential to provide a comprehensive perspective on the myriad areas of concerns in oncology.

Although the ICF has historically been used most frequently in a rehabilitation-based context, ${ }^{8}$ it has been utilized for a diverse array of purposes in the field of oncology, including: evaluating functioning in individuals with cancer; ${ }^{9-11}$ guiding assessment in oncology rehabilitation; ${ }^{12-14}$ assessing the comprehensiveness of outcome measures utilized in oncology research; ${ }^{15-17}$ assisting in health-related QOL instrument selection; ${ }^{16,18,19}$ and comparing the primary concerns of health professionals with those of their patients. ${ }^{20}$ In fact, use of the ICF in oncology has become so pervasive that the International Psycho-Oncology Society recently called for the "development of assessment instruments focusing on aspects of the ICF," ${ }^{21}$ such as functional consequences related to psychosocial activities and participation. These observations reinforce the importance of the ICF to cancer-related research and practice.

Perhaps more important than how the ICF has been used in oncology is why it may be of benefit. Recognizing that cancer-related concerns extend beyond just curing the malignancy, the field of oncology has developed several models that emphasize one particular area of concern, such as psychosocial issues or the delivery of care. However, these models often fail to address the full range of concerns that face an individual with cancer. Instead, the ICF offers a broader and better alternative to the currently available frameworks. First, and owing to its underlying principles, the ICF aims to serve as a neutral, comprehensive, and universally applicable framework of health and functioning. Unlike other models of health in oncology, the ICF directly promotes and facilitates interdisciplinary collaboration through its common terminology for classifying health and its related states, determinants, and outcomes. This unified language enhances communication among practitioners, researchers, policy makers, and the general public. The ICF's success in facilitating interdisciplinary collaboration is evident by the fact that the ICF has been utilized by numerous health professional groups who work collaboratively in oncology, including nurses,${ }^{8}$ physiotherapists, ${ }^{22}$ psychologists, ${ }^{15}$ and speech-language pathologists. ${ }^{12,13}$ The ICF is also being actively integrated into undergraduate medical programs, ${ }^{23}$ occupational therapy training programs, ${ }^{24}$ and other 
graduate-level health education programs, ${ }^{25}$ thus ensuring its continued presence in the field of health care.

One distinct advantage of the ICF is that it can be adapted to account for multiple comorbidities and varying states of health and illness. With revisions, it can also account for issues throughout the continuum of care including issues that arise in survivorship. While the ICF does not propose to be a model for the delivery of health care, it does provide a common ground to facilitate interdisciplinary collaboration and recognition of a broad range of physical, psychosocial, and practical concerns. These concerns, once formally acknowledged through use of the ICF, can be addressed in clinical settings through use of an existing care delivery model. Ultimately, in order to better facilitate interdisciplinary collaboration, oncology requires a unified framework of health that transcends disciplinary boundaries and priorities to describe one's health state throughout the entire continuum of care. With some adaptations, the ICF has the potential to provide a biopsychosocial perspective of care that captures the diverse array of concerns throughout the entire continuum of cancer care.

Unfortunately, and despite many strengths of the ICF, in its current form some key conceptual shortcomings exist; specifically, it is limited in its ability to address the heterogeneity of concerns related to cancer. To demonstrate this belief, a brief discussion of the primary areas of concern for oncology in which the ICF exhibits conceptual limitations will follow. Specifically, the discussion to follow will center on the proposed revision of the following three areas of the ICF framework: (1) the replacement of the term "health condition" with the more inclusive and dynamic term "health state;" (2) the continuing development and refinement of the personal factors component to ensure issues such as comorbidities can be accounted for appropriately; and (3) the inclusion of a mechanism to account for the subjective dimension of health and functioning (eg, QOL). It is through the expansion of these conceptual parameters that the ICF may become more relevant and applicable to the field of oncology. With these important revisions, the ICF has the potential to provide a broader biopsychosocial perspective of care that captures the diverse range of concerns that arise throughout the continuum of care in oncology.

\section{Area of concern - terminology: health condition versus health versus health state}

Presently, the ICF includes consideration of an individual's health condition (eg, disorder or disease), functional state (eg, body functions and structures, activities, participation) and contextual factors (eg, environmental and personal factors). "Health condition" is defined by the ICF as: "an umbrella term for disease (acute or chronic), disorder, injury, or trauma. A health condition also may include other circumstances such as pregnancy, aging, stress, congenital anomaly, or genetic predisposition." Since the overall aim of the ICF is to provide a common language and framework for the description of "health and health-related states," ${ }^{\text {it seems }}$ somewhat contradictory to have the conceptual framework situated according to an individual's health condition. Such a position may limit a comprehensive understanding of the individual's health-related experience. Consequently, replacing the term "health condition" with a more inclusive alternative may help to better represent the health-related experience.

Expanding on this idea of replacing "health condition" with a more inclusive term, it is useful to examine previous efforts to address this issue. In particular, one such effort undertaken by Dufour and Lucy proposed replacing "health condition" with "health," which they envisioned as the outcome of care that illustrates health status from the perspective of the patient, family, and practitioners. ${ }^{26}$ Similarly, Huber et al augmented this health-focused view to situate the terms "health condition" and "health state" as components of an individual's overarching health. ${ }^{27}$ This approach to health is more in line with a universal perspective, however, the emphasis on the term "health," as defined by the $\mathrm{WHO},{ }^{28}$ remains problematic. While "good health" is likely the goal of all individuals, the attainment of "a state of complete physical, psychological, and social wellbeing" ${ }^{28}$ is neither practical nor feasible, particularly for individuals diagnosed with cancer. Although it is important to acknowledge that the WHO's definition of health substantially altered the way health is viewed by acknowledging that disease and infirmity are insufficient to describe health in the absence of the subjective experience, the definition is difficult to operationalize. ${ }^{29}$

The difficulty in utilizing the terms "health" and "health condition" according to WHO definitions is particularly evident in oncology. To illustrate this point, a brief case example is appropriate:

A man is diagnosed with laryngeal cancer and is subsequently scheduled to undergo radiotherapy treatments. Prior to treatment, his health condition would be classified as "laryngeal cancer." In terms of health, he would be classified as "unhealthy" given his diminished physical state. Additionally, the specific domains of the ICF (eg, body 
functions and structures, activities, participation, contextual factors) could account for any diminished functioning resulting from the health condition. During radiotherapy, the health condition may still be present; however, his healthrelated experience is likely to change depending on how he responds to the side effects of the treatment. Depending on the experienced intensity of his symptoms, his levels of activity and participation, and therefore his levels of functioning and disability, are likely to change. However, throughout all of this change, his "health condition" and lack of "health" remain the same according to the current ICF. As time proceeds and he completes his treatment and eliminates the cancer, additional concerns arise. The health condition (eg, laryngeal cancer) has been eliminated; however, he is left with significant physical and psychological consequences of his disease and its treatment. Since he is no longer in possession of a "health condition" because his cancer has been eliminated, his persistent physical and psychological concerns cannot be properly accounted for and classified according to the criteria of the ICF.

As a classification system, the "ICF systematically groups different domains for a person in a given health condition." 6 Consequently, use of the term "health condition" in the ICF is insufficient and problematic because it limits a full understanding of an individual's health-related experience. This is because the ICF cannot account for concerns that persist after the health condition has been eliminated biologically. Moreover, use of the static terms "health" or "health condition" to classify the health-related experience of an individual may fail to recognize dynamic changes in functioning and overall wellbeing. Instead, the term "health state" may serve as a more appropriate alternative to "health" or "health condition" for the purpose of classifying one's health-related experience.

In order to address some of the limitations described above, a revised definition of the term "health state" is proposed as follows:

A dynamic continuum of health-related functioning and perceived wellbeing that can account for varying healthrelated phases of an individual's life. The continuum of health state may range from poor to excellent and may include consideration of multiple health conditions at one time.

Thus, health state represents a dynamic continuum of functioning, in contrast to "health" or "health condition" which represent static entities. In essence, one is either healthy or unhealthy; in possession of a health condition or free of a health condition. In reality, it is unlikely that an individual could remain in a stable state of health if evaluated according to the WHO definition. However, as defined above, "health state" is a variable, contingent status, which is particularly relevant to oncology. Replacing the term "health condition" with "health state" in the ICF will allow sound exploration of an individual's health-related functioning and wellbeing throughout varying disease states, such as throughout the continuum of care in oncology where an individual may transition from a state where the neoplastic condition is present (eg, following diagnosis, during treatment) to a state where the neoplastic condition has been eliminated (eg, survivorship). Additionally, the consequences of treatment may persist as a negative health state. Thus, approaching one's health-related state from a dynamic perspective will allow the ICF to fulfill its goal of ensuring that "universality [is] a major aim." A system that permits exploration of one's health status across a continuum of health states (ranging from poor to excellent health), is one that is aligned with a universal perspective. Moreover, a framework that acknowledges that one's health state is a dynamic and varying phenomenon that can be influenced by the consequences of treatment is one that will be capable of capturing a wide range of concerns throughout all stages of the continuum of care in oncology.

\section{Considerations throughout the continuum of care}

When considered in the context of oncology, it is apparent that the term "health condition" cannot be applied throughout all stages of care. The continuum of care in oncology begins with one's initial diagnosis and proceeds to treatment, completion of treatment, rehabilitation (if required) and survivorship, or palliation and death. ${ }^{30}$ For the purposes of this treatise, survivorship is defined as the "period in a cancer patient's life, which is posttreatment, separate from diagnosis and treatment and from end-of-life care." ${ }^{\text {31 }}$ While the term "health condition" may be appropriate when applied to one's state during diagnosis or treatment, it does not extend to states that remain when the cancer itself has been eliminated, such as in survivorship. In a review of QOL among long-term (10-year) head and neck cancer survivors, Mehanna and Morton determined that at 10 years following diagnosis, participants' overall QOL declined significantly by an average of $11 \%$ compared to before treatment. ${ }^{32}$ Additionally, QOL declined by $15 \%$ when compared with postdiagnostic QOL at years one and two. Interestingly, none of the sociodemographic, disease- (eg, health condition), or treatment-related variables 
predicted long-term QOL, which implies that there may be a unique phenomenon present in cancer survivorship that causes ongoing concerns but is distinct from one's initial health condition. This idea suggests that "health condition" is not conducive to a universal model of health (on which the ICF purports to be founded), since a truly universal model of health would permit exploration of one's health state regardless of the presence or absence of a diagnosable "health condition."

Essentially, cancer survivorship highlights the fact that an individual's health state can be compromised, without the presence of an emergent and/or unique health condition. This phenomenon is evident in the examination of both long-term and late effects of cancer. Long-term effects (eg, chronic or persistent problems) consist of adverse consequences that arise during treatment and continue beyond the completion of treatment. ${ }^{33}$ Examples may include cancer-related fatigue, cognitive or sexual difficulties, changes in body image, elevated anxiety or depression, and neuropathies with consequent weakness, numbness, or pain..$^{34,35}$ Alternatively, late effects of cancer and its treatment are generally defined as problems that are absent or unidentified at the end of treatment, but may develop as outgrowths of the effects of treatment on organ systems or the psychological process. With concerns such as depression, the distinction between its presence as a late versus long-term effect may be challenging to determine. However, in other instances, such as with musculoskeletal complications or late-onset stamina deficits related to thyroid or cardiovascular concerns, the distinction may be more pronounced and disabling, since the presence of a symptom such as those described above may signal an emerging medical problem. ${ }^{34}$ Since it is evident that an individual's health state can be negatively impacted by cancer or its treatment several years after completing treatment, it is unfortunate that the ICF does not permit exploration of these concerns in its current format.

The incomplete nature of the ICF in acknowledging and properly classifying the elevated burden on cancer survivors suggests that the impact of late and long-term effects on functioning and overall outcomes cannot be accounted for in a meaningful manner. This is problematic given that a population-based comparison of cancer survivors with matched controls found a substantially increased burden of illness in cancer survivors, manifested in days lost from work, inability to work, poor general health perception, and the need for help with daily activities. ${ }^{36}$ Furthermore, compared with age-matched controls, cancer survivors reported poorer health outcomes, decreased functioning, and higher levels of burden across multiple domains. Interestingly, these decrements were consistent across tumor sites and time since diagnosis. ${ }^{36}$ Additionally, these concerns and functional decrements appear to persist across age categories..$^{37,38}$ These data suggest that, in general, cancer survivors experience an elevated burden of illness compared to those without cancer. Further, this relationship appears to exist irrespective of age, tumor site, or time since diagnosis. As such, it would seem important to classify the healthrelated status of individuals with cancer once the cancer has been eliminated and they enter survivorship.

\section{Addressing the impact of comorbidities on health state}

In addition to concerns that arise in survivorship, the term "health condition" presents an additional challenge. Use of the singular state of the word "condition" suggests that the ICF is not designed to explore comorbidities, which are frequently present in oncology. ${ }^{39}$ This singular terminology has the potential to be problematic given that comorbidities may exist prior to the diagnosis of cancer (eg, diabetes, hypertension, scleroderma) or emerge as a result of treatment. Understanding the interaction between cancer and comorbidities is vital to clinical care because comorbidities can profoundly influence many aspects of care including: prevention, screening, diagnosis, prognosis, treatment, and ongoing health service needs. ${ }^{40}$ Furthermore, when comorbidities are considered in conjunction with survivorship, additional concerns arise. In a study of older adult $(\geq 60)$ long-term cancer survivors, the number of current comorbidities was deemed to be the best predictor of functional difficulties and also a significant predictor of participation restriction(s). ${ }^{38}$ Additionally, survivors of cancer with comorbid conditions have been shown to fare worse than survivors without documented comorbidities. ${ }^{41}$ Despite the documented relationship between comorbidities and functioning, the current ICF cannot account for their role due to the current definition of personal factors and the designation of comorbidities as personal factors. Furthermore, it cannot account for the impact of comorbid health concerns in survivorship. Consequently, a revision of terminology is recommended in order to facilitate a broader perspective of one's health-related experience.

\section{Recommendation one: replace "health condition" with "health state"}

A key recommendation for revision of the ICF to make it more applicable to oncology is replacement of the term 
"health condition" with "health state." This change will ensure that all individuals, regardless of their location on the cancer continuum (eg, survivors), can be included in the classification. It will also promote the universal approach to classification advocated for by the ICF creators. Furthermore, it may facilitate understanding that the cancer experience extends beyond just the diagnosis and treatment phases of disease and that one's health state may still be in flux at that time. Essentially, the "health condition" is just one facet of the disease experience. The term "health state" permits a broader perspective on the dynamic experience of health and accounts for the changes in health status in a meaningful manner.

\section{Area of concern: development of the personal factors component}

In the current ICF framework, personal factors consist of the features of an individual that are "not part of a health condition or health state." According to the ICF, these features may include age, race, gender, lifestyle factors, habits, coping mechanisms, social background, education, profession, behavior patterns, individual psychological assets, and other health conditions, among other factors. While this list may appear broad in scope, in fact, the personal factors section is the least developed component of the ICF. Unlike the other elements of the ICF (with exception to the "health condition"), the personal factors component has no taxonomical classification. The lack of formal classification may create the impression that personal factors are not as important as other components of the ICF. Despite this potential misconception, personal factors are a vitally important area of consideration in oncology. Relative to one's cancer-related experience, personal factors have the potential to profoundly influence an individual's morbidity, ${ }^{42}$ risk factors, ${ }^{43,44}$ functioning, ${ }^{45}$ QOL, ${ }^{46,47}$ and decision making regarding treatment options. ${ }^{48}$ As such, special attention must be paid to the revision and expansion of this critically important area of the ICF in order to ensure that the ICF is able to account for factors of importance and relevance to the field of oncology.

\section{Influence of personal factors on health state}

Although the ICF is a classification of health and healthrelated states, the personal factors component is comprised of features of an individual that are not part of the health condition or health state. This means that the ICF does not classify socioeconomic factors (eg, gender, race), other health conditions, or individual features which are not related to health, as personal factors. ${ }^{49}$ While some authors have noted that a health condition can be strongly related to a personal factor such as gender (eg, ovarian cancer) while not being directly caused by it, ${ }^{27}$ there are instances where personal factors have been shown to cause or exacerbate health conditions. For instance, excess body weight (attributed to lifestyle choices, habits, and health behaviors, all of which are personal factors according to the ICF) has been shown to increase the risk of developing cancer at several sites including the esophagus, kidney, colon, prostate, thyroid, pancreas, and postmenopausal breast. ${ }^{50}$ Additionally, both socioeconomic status and race have been tied to inequities in quality of care and survival in oncology. ${ }^{51}$ Further, the manner in which one is able to cope and adjust to their cancer has been shown to influence QOL ${ }^{46,47}$ and both physical and psychological functioning. ${ }^{45}$ Due to these established relationships between personal factors and health state, personal factors cannot be considered in isolation of one's health state and failure to meaningfully address the relationship between one's health state and personal factors directly limits the utility of the ICF in cancer care.

\section{Personal factors and oncology}

In the realm of oncology, personal factors offer a valuable contribution with respect to the determination of risk factors, contribution to morbidity, and decision making by both patients and practitioners. Specifically, with regard to risk factor information, personal factors such as: age, race, gender, lifestyle factors, habits, behavioral patterns, and other health conditions, contribute significantly to established risk factors for a variety of cancer sites. For example, both reproductive history and medical history serve as risk factors for breast cancer, ${ }^{43}$ whereas smoking status, exposure to secondhand smoke, air pollution, dietary factors, alcohol intake, level of physical activity, ${ }^{52}$ reproductive factors, and hormone use ${ }^{53}$ are known risk factors for lung cancer. Similarly, smoking status, alcohol intake, diet, oral hygiene, genetic predisposition, preexisting medical conditions, and infectious agents such as the Epstein-Barr virus or the human papillomavirus ${ }^{44}$ represent common risk factors for head and neck cancer. While the range of risk factors for these cancer sites is broad, all of these factors are considered personal factors according to the current ICF framework. The problem with this relates to the fact that personal factors are not classified in the current ICF, which means that they cannot be properly accounted for by users of the ICF framework.

The importance of classifying personal factors is apparent when examining the relationship between personal factors and 
morbidity in cancer survivorship. For instance, personal factors can contribute to decreased morbidity in survivors of cancer. ${ }^{42}$ This is particularly evident in factors related to modifiable lifestyle factors such as alcohol intake, dietary choices, and level of exercise, but is also apparent in unmodifiable personal factors such as gender, race, age at diagnosis, and health problems preceding the cancer diagnosis. ${ }^{42}$ Understanding the role of personal factors in cancer care is important because modifiable factors have the potential to reduce health risks associated with cancer and often represent the primary method of risk reduction available to cancer survivors. ${ }^{42}$

The influence of personal factors on decision making by both individuals with cancer and their practitioners should also not be overlooked. For example, a qualitative study into the factors influencing treatment decisions made by men undergoing treatment for prostate cancer determined that a dialogue which addresses personal factors (eg, age, profession, health status, personality traits, personal lifestyle choices, life experiences, personal philosophy, spiritual beliefs, ethnicity, potential family burden, QOL) in addition to medical factors can guide men towards making the best informed decision for their care of localized prostate cancer. ${ }^{48}$ Furthermore, a study examining practices of speech-language pathologists determined that consideration of unmodifiable personal factors, such as age, gender, ethnicity, and previous experiences, allowed practitioners to better understand their clients and adapt services to meet their needs accordingly. ${ }^{54}$ In essence, acknowledgment of the vital role of personal factors resulted in both better informed decision making among patients and the provision of highly tailored care from practitioners. Both of these factors contribute to an environment where patient-centered care can flourish.

\section{Influence of personal factors on patient-centered care}

Patient- or client-centered care is a hallmark of comprehensive cancer care; it is defined as care that:

(a) explores the patients' main reason for the visit, concerns, and need for information; (b) seeks an integrated understanding of the patients' world - that is, their whole person, emotional needs, and life issues; (c) finds common ground on what the problem is and mutually agrees on management; (d) enhances prevention and health promotion; and (e) enhances the continuing relationship between the patient and the doctor. ${ }^{55}$

Oncology care utilizes a patient-centered care model, which implies that optimal health care delivery requires information that extends beyond the scope of body functions, structures, activities, and participation. ${ }^{56}$ Specifically, in order to engage in effective patient-centered care, personal factors such as an individual's past and present experiences, coping style, self-efficacy, attitudes, values, preferences, and knowledge are relevant factors for consideration. ${ }^{57}$ As such, utilizing patient information that would be considered "personal factors," according to the ICF, is necessary in order to understand the needs and desires of the individual and subsequently provide personalized care. Accordingly, clarifying the language regarding both the definition and description of personal factors in the ICF would help to eliminate ambiguities and better align the ICF with the objectives of patient-centered care.

\section{ICF contradictions in the definition and description of personal factors}

While an aim of the ICF was to provide a common terminology for the description of health and its related states, ${ }^{6}$ there are some inherent inconsistencies in the terminology used in the ICF. Specifically, there is a contradiction between both the definition and description of personal factors. According to the definition stated in the ICF, comorbidities are not viewed as personal factors, whereas in the description, comorbidities are viewed as personal factors. ${ }^{6}$ If the definition is correct, then it appears that the ICF is designed to address only one health-related concern at a time (eg, the primary neoplasm). Conversely, if the description is correct, then it seems as though comorbidities have been assigned to a category where a precise description of their disease-related sequelae is not addressed. In order to remedy this inconsistent position regarding comorbidities, it may be helpful to adopt an alternative definition of health state that specifically accounts for coexisting health conditions, such as the one proposed earlier in this paper.

\section{Recommendation two: continue the development and refinement of personal factors}

A second recommendation for revision of the ICF in an effort to enhance its relevance to oncology is the development and refinement of the personal factors component through structured, taxonomical classification. While the goal of "development of a personal factors component" 6 is shared by the ICF creators and ICF researchers alike, recommendations for its achievement are not stipulated in the ICF. Undoubtedly, the taxonomical development of the personal factors components of the 
ICF will be a challenging task due to the diverse range of potential items. It is, however, an important step to undertake if personal factors are to be considered equally with other ICF components. Efforts to initiate the process of developing a taxonomy for the classification of personal factors through a systematic review and content analysis of existing literature have been recently reported. ${ }^{56}$ While this process is in its infancy, it is an important first step in the further development of the personal factors component of the ICF. Therefore, it is imperative that efforts such as this continue. Conducting consultations with specialists (including those with illnesses and impairments) from a wide variety of fields, including oncology, will help to ensure that a broad range of perspectives are considered and included. These efforts may subsequently help ensure that a comprehensive perspective on the components that constitute personal factors are identified and included in the taxonomy. Further, it is recommended that the role of comorbidities be clarified and assigned a position in the classification system. These modifications will ensure that important personal factors that influence an individual's health state can be accounted for in the ICF.

\section{Area of concern: accounting for the subjective dimension of health and functioning}

The ability to understand and account for an individual's subjective experience of their health and functioning (eg, QOL) is vital to the provision of patient-centered care in oncology. This idea of attending to one's subjective experience is also well aligned with a biopsychosocial perspective of health. Given that the ICF is informed by a biopsychosocial perspective, it would seem reasonable to assume that both the ICF and assessments of QOL would closely align with one another. However, there is a key distinction between the concept of QOL and the conceptual foundation of the ICF that limits this assumption, ie, the ICF is based on the objective assessment of functioning and contextual factors, whereas QOL is by definition a subjective construct. In essence, QOL cannot be accounted for solely by the objective measurement and classification of functioning. Yet, the current ICF is structured in such a way that it cannot account for the subjective experience of health and functioning in either its coding system or conceptual framework.

The WHO defines QOL as:

An individual's perception of their position in life, in the context of their culture and values system where they live, and in relation to their goals, expectations, standards, and concerns. It is a broad ranging concept, incorporating in a complex way a person's physical health, psychological state, level of independence, social relationships, personal beliefs, and relationship to salient features of the environment. ${ }^{58}$

Despite the fact that the WHO developed both the ICF and this definition of QOL, there remains a sharp disconnect in the health-related values espoused by these WHO documents. If QOL is indeed a broad ranging concept that incorporates an individual's "physical health, psychological state ... social relationships ... and relationship to salient features of the environment," ${ }^{58}$ then it would be reasonable to assume that comprehensive evaluations of QOL would be consistent with the biopsychosocial model of health which values these same aspects and theoretically underpins the ICF. Moreover, given that the ICF is able to objectively classify components of the physical, psychological, social, and environmental domains of an individual's life, one may raise the question as to why the explicit inclusion of QOL in the ICF is a contentious subject.

Proponents of the ICF have addressed this QOL debate through three primary arguments. The first consists of the claim that the categories of the ICF which comprise functioning (eg, body functions and structures, activities, participation) can serve to operationalize QOL. ${ }^{59}$ However, this interpretation is in contradiction to the WHO's definition of QOL as a subjective construct; it fails to account for the influence of an individual's personal goals, expectations, standards, and concerns - all of which are essential to understanding an individual's QOL. The second argument points to the fact that the ICF document describes itself as a research tool "to measure outcomes, QOL, or environmental factors." However, despite this assertion by the ICF creators, QOL has been omitted from both the conceptual framework and the classification system. The fact that the ICF document indicates that it can be used to measure QOL - considered in conjunction with the exclusion of QOL from the framework and coding system - suggests that ICF users should be able to infer one's QOL from descriptions of their functioning according to the existing components of the ICF. But as noted by Huber et al, two individuals with the same health condition may experience similar situations very differently due to the meaning that they place on certain activities and/or levels of participation. ${ }^{27}$ This suggestion that QOL can be inferred by objective measures is reinforced by the third argument made by proponents of the ICF, which is that the ICF can serve as a "gold standard" for ensuring the comprehensiveness of health-related QOL measures. ${ }^{60}$ 
This argument in favor of using the ICF as a benchmark for evaluation of health-related QOL measures is based on the assertion that "a content comparison based on a universally accepted, well-defined and standardized reference system, that allows for a detailed exploration and comparison of all contents of the measures would be valuable." ${ }^{100}$ While this point is accurate, in its present form the ICF cannot serve as an appropriate benchmark for evaluating an individual's subjective perception of their QOL. As noted by the authors of the content comparison, $12 \%$ of the items from the QOL measures could not be represented by the ICF categories. ${ }^{60}$ Specifically, these items related to both subjective perceptual concerns (eg, satisfaction, QOL) and unclassified personal factors. Despite the numerous similarities between QOL measures and the ICF, there remains a fundamental incompatibility between the concept of QOL and its operationalization according to the ICF.

Specifically, for Geyh et al to argue that QOL can be inferred from one's objective functional status demonstrates a misunderstanding of the fundamental concept of QOL. ${ }^{60}$ Functioning and QOL, while not mutually exclusive, are fundamentally distinct concepts according to their definitions. As noted by Cruice, the functioning components of the ICF framework outline what an individual can and cannot do, whereas QOL permits us to consider who an individual is and what he or she wants in life.$^{61}$ Consequently, to assume that one can infer what an individual perceives to be an acceptable level of QOL based on what they are capable of doing is to underestimate that individual as a whole person - with thoughts, perceptions, values, and desires. As a result, this information must be gathered directly from the individual in question.

Evidence for the subjectivity of QOL is apparent in a study of individuals undergoing autologous peripheral blood stem cell transplantation where researchers found that patient-perceived QOL was primarily dependent on family and social interactions, not health and physical functioning as researchers had anticipated. ${ }^{62}$ This finding was supported by Moons et al, who determined that health care practitioners focus primarily on objective aspects related to physical functioning, whereas those receiving care report more subjective concerns related to psychosocial wellbeing and QOL. ${ }^{63}$ Undoubtedly, both objective and subjective concerns provide unique and important information on one's status; however, clinicians must consider both forms of information when planning a comprehensive, yet individualized program of care. As such, if the ICF is to be utilized by oncology professionals, it must be able to account for both objective and subjective concerns. If subjective concerns (eg, one's ability to cope) exist, they cannot be overlooked or undervalued in lieu of more tangible (and perhaps more easily measured) physical concerns solely because practitioners may be more comfortable dealing with physical functioning. This is particularly true in oncology where psychosocial and QOL concerns are common.

\section{QOL and cancer care}

Attentiveness to QOL issues by health care team members is essential to the provision of effective and comprehensive care in oncology. Ensuring the wellbeing of those with cancer requires the acknowledgment that an individual's QOL may be influenced by multidimensional factors. For instance, the burden of cancer is often largely manifested in psychosocial dysfunction, which can negatively impact one's perceived QOL. ${ }^{3}$ As a result, one of the primary benefits to understanding the variation in an individual's QOL is to minimize the negative impact of cancer on one's life. ${ }^{64}$ Understanding the relationship between QOL and modifiable psychosocial factors may permit tailored interventions to be constructed with the goal of maximizing individual QOL.

Table I Summary of key concerns and recommendations

\begin{tabular}{|c|c|c|}
\hline Area of inquiry & Key concern & Recommendation \\
\hline Health condition versus & "Health condition" is a singular, static term that limits a comprehensive & Replace the term "health condition" \\
\hline \multirow{2}{*}{ health state } & understanding of an individual's health-related experience, particularly & with the more inclusive and \\
\hline & as it relates to comorbidities and survivorship concerns in oncology. & dynamic term "health state." \\
\hline \multirow[t]{4}{*}{ Personal factors } & Despite the significant influence of personal factors on oncology, & Continue to develop and refine \\
\hline & the ICF does not provide a taxonomical classification for the personal & the personal factors component to \\
\hline & factors domain. Further, there is an inherent contradiction between & ensure issues such as comorbidities \\
\hline & the definition and description of personal factors. & can be accounted for appropriately. \\
\hline \multirow[t]{3}{*}{ QOL } & Because the ICF is based on the objective assessment of functioning & Include a mechanism in the ICF \\
\hline & and contextual factors, it cannot account for the subjective & to account for the subjective \\
\hline & experience of health (eg, QOL). & $\begin{array}{l}\text { dimension of health and functioning } \\
\text { (eg, QOL). }\end{array}$ \\
\hline
\end{tabular}

Abbreviations: ICF, International Classification of Functioning, Disability, and Health; QOL, quality of life. 
Ongoing assessment of the dimensions of QOL has the ability to provide valuable information regarding the longterm outcomes of cancer treatment and its associated side effects. ${ }^{65}$ Information obtained through such assessments can then be utilized to identify areas where rehabilitation and additional support may be required and subsequently guide the appropriate psychosocial interventions with the goal of improving QOL.

In addition to identifying the need for psychosocial interventions, data related to QOL have also been shown to predict survival in individuals with cancer. ${ }^{66,67}$ For instance, a meta-analysis conducted by Chida et al demonstrated that stress-related psychosocial factors were associated with poorer head and neck cancer survival. ${ }^{66}$ Additionally, the increasing incidence of cancer, ${ }^{68}$ considered in conjunction with the improving rates of survival, ${ }^{69}$ suggests that evaluations of QOL in oncology will remain important as individuals adjust to the challenges of not only active treatment, but also survivorship. Fundamentally, QOL is a key component of compassionate care and its acknowledgment continues to grow in oncology.

Professional recognition of the importance of QOL measures in oncology is evident in the decision of the US Food and Drug Administration to require data on healthrelated QOL as a basis for the approval of new anticancer drugs. ${ }^{70}$ This formal regulation considered in conjunction with the increased use of health-related QOL measures by clinicians, reflects a professional awareness that the individual's perspective is at the core of decisions related to health care provision and research in oncology. In essence, it is the critical subjective dimension of individual perception and preferences that characterizes QOL as a distinct concept that is worthy of acknowledgement in a comprehensive framework of health, such as the ICF.

\section{Recommendation three: accounting for the subjective dimension of health and functioning}

A third recommendation for revision of the ICF to increase its relevance to the field of oncology is the addition of the subjective dimension of health and functioning, or QOL. If the ICF can be adapted to include QOL, it will present a much more comprehensive and suitable option to guide care in oncology. Whether QOL is included in the form of an emergent and unique factor, ${ }^{71,72}$ an overarching factor, ${ }^{73}$ or as a contributing factor to an existing component of the $\mathrm{ICF},{ }^{27}$ the key matter of importance is that it is included as an integral part of the ICF. Without explicit acknowledgment of the relationship between one's subjective cancer experience and their objective functioning, the ICF will remain conceptually limited as a framework for use in oncology care. Yet the inclusion of this critical linkage may be facilitated if the attributes that characterize both subjective and objective indices of health specific to cancer care can be accepted as essential constructs of the human condition.

\section{Summary}

This treatise has explored the ICF with a focus on comprehensive cancer care. Oncology offers a unique lens from which to examine the structure of the ICF and the continuum of care that is ideally provided to individuals with cancer. Ultimately, from the perspective of oncology, this framework is viewed as a valuable tool for guiding patient care, but it currently possesses some areas of limitation that necessitate conceptual revision; to this end, several recommendations have been proposed.

Unlike other frameworks of health, the ICF facilitates interdisciplinary collaboration through use of common terminology, which enhances communication across disciplines. With revision, the ICF could account for multiple comorbidities and varying states of health, and importantly, could account for issues encountered throughout the continuum of care including concerns that arise in survivorship. While the ICF does not present itself as a model for the delivery of health care, it does provide a common ground to facilitate interdisciplinary collaboration and recognition of a broad range of physical, psychosocial, and practical concerns. These concerns, once formally acknowledged through use of the ICF, can be addressed in clinical settings through use of an existing care delivery model in conjunction with open discussions about individual preferences. Optimal cancer care requires a unified framework of health that transcends disciplinary boundaries in an effort to describe one's heath state and subjective concerns throughout the entire continuum of care. With revisions as noted herein, the ICF has the potential to embody a true biopsychosocial perspective of care. Through such consideration and revision, the ICF may then more appropriately capture the diverse range of concerns that arise throughout the continuum of care in oncology. The result of such efforts may be realized with the ultimate outcome being enhanced sensitivity and acknowledgment of the impact of cancer on those who experience this disease, members of their family, and those who are charged with their care.

\section{Acknowledgments}

$\mathrm{CB}$ is the recipient of a Doctoral Research Award from the Canadian Institutes of Health Research. 


\section{Disclosure}

The authors report no conflicts of interest in this work. This manuscript was developed as a component of one of the author's (CB) comprehensive exam process. Thus, it was conceived of and subsequently developed through an iterative process with the coauthors. As such, it may be viewed as a literature review that aimed to examine the ICF framework through an oncologic perspective.

\section{References}

1. World Health Organization. The Global Burden of Disease: 2004 Update. Geneva: World Health Organization; 2008. Available from: http://www.who.int/healthinfo/global_burden_disease/GBD_ report_2004update_full.pdf. Accessed January 24, 2013.

2. Jemal A, Bray F, Center MM, Ferlay J, Ward E, Forman D. Global cancer statistics. CA Cancer J Clin. 2011;61(2):69-90.

3. Semple CJ, Sullivan K, Dunwoody L, Kernohan WG. Psychosocial interventions for patients with head and neck cancer: past, present, and future. Cancer Nurs. 2004;27(6):434-441.

4. Aziz NM. Radiation biocontinuum: follow-up care for cancer survivors: needs, issues, and strategies. In: Rubin P, Constine LS, Marks LB, Okunieff P, editors. Late Effects of Cancer Treatment on Normal Tissues. Berlin: Springer-Verlag; 2008:109-130.

5. Montazeri A. Health-related quality of life in breast cancer patients: a bibliographic review of the literature from 1974 to 2007. J Exp Clin Cancer Res. 2008;27(1):32.

6. World Health Organization. The International Classification of Functioning, Disability and Health. Geneva: World Health Organization; 2001.

7. Engel GL. The need for a new medical model: a challenge for biomedicine. Science. 1977;196(4286):129-136.

8. Kearney PM, Pryor J. The International Classification of Functioning, Disability and Health (ICF) and nursing. $J$ Adv Nurs. 2004;42(6):162-170.

9. Ajovalasit D, Vago C, Usilla A, et al. Use of ICF to describe functioning and disability in children with brain tumours. Disabil Rehabil. 2009;31(Suppl 1):S100-S107.

10. Tschiesner U, Linseisen E, Baumann S, et al. Assessment of functioning in patients with head and neck cancer according to the International Classification of Functioning, Disability, and Health (ICF): a multicenter study. Laryngoscope. 2009;119(5):915-923.

11. Wright $M$. Children receiving treatment for cancer and their caregivers: a mixed methods study of their sleep characteristics. Pediatr Blood Cancer. 2011;56(4):638-645.

12. Eadie TL. The ICF: a proposed framework for comprehensive rehabilitation. Am J Speech Lang Pathol. 2003;12(2):189-197.

13. Eadie TL. Application of the ICF in communication after total laryngectomy. Semin Speech Lang. 2007;28(4):291-300.

14. Gilchrist LS, Galantino ML, Wampler M, Marchese VG, Morris GS, Ness KK. A framework for assessment in oncology rehabilitation. Phys Ther. 2009;89(3):286-306.

15. Becker S, Kirchberger I, Cieza A, et al. Content validation of the Comprehensive ICF Core Set for Head and Neck Cancer (HNC): the perspective of psychologists. Psychooncology. 2010;19(6):594-605.

16. Tschiesner U, Rogers SN, Harreus U, Berghaus A, Cieza A. Content comparison of quality of life questionnaires used in head and neck cancer based on the International Classification of Functioning, Disability and Health: a systematic review. Eur Arch Otorhinolaryngol. 2008;265(6):627-637.

17. Brockow T, Duddeck K, Geyh S, et al. Identifying the concepts contained in outcome measures of clinical trials on breast cancer using the International Classification of Functioning, Disability and Health as a reference. $J$ Rehabil Med. 2004;(Suppl 44):43-48.
18. Fayed N, Schiariti V, Bostan C, Cieza A, Klassen A. Health status and QOL instruments used in childhood cancer research: deciphering conceptual content using World Health Organization definitions. Qual Life Res. 2011;20(8):1247-1258.

19. Cieza A, Stucki G. Content comparison of health-related quality of life (HRQOL) instruments based on the International Classification of Functioning, Disability and Health (ICF). Qual Life Res. 2005;14(5): $1225-1237$.

20. Tschiesner U, Becker S, Cieza A. Health professional perspective on disability in head and neck cancer. Arch Otolaryngol Head Neck Surg. 2010;136(6):576-583.

21. Koch U. IPOS Sutherland Memorial Lecture: psycho-oncology and health care research. Psychooncology. 2008;17(6):533-537.

22. Glaessel A, Kirchberger I, Stucki G, Cieza A. Does the Comprehensive International Classification of Functioning, Disability and Health (ICF) Core Set for Breast Cancer capture the problems in functioning treated by physiotherapists in women with breast cancer? Physiotherapy. 2011;97(1):33-46.

23. Gutenbrunner C, Schiller J, Schwarze M, et al. Hannover model for the implementation of physical and rehabilitation medicine teaching in undergraduate medical training. $J$ Rehabil Med. 2010;42(3):206-213.

24. Cockburn L, Tretham B, Kircsh B. Educating tomorrow's practitioners: influences on community mental health occupational therapy education. Bull World Fed Occup Ther. 2005;52:16-24.

25. Reed GM, Dilfer K, Bufka LF, et al. Three model curricula for teaching clinicians to use the ICF. Disabil Rehabil. 2008;30(12-13):927-941.

26. Dufour SP, Lucy SD. Situating primary health care within the International Classification of Functioning, Disability and Health: enabling the Canadian Family Health Team initiative. J Interprof Care. 2010;24(6):666-677.

27. Huber JG, Sillick J, Skarakis-Doyle E. Personal perception and personal factors: incorporating health-related quality of life into the International Classification of Functioning, Disability and Health. Disabil Rehabil. 2010;32(23):1955-1965.

28. World Health Organization. Constitution of the World Health Organization: Basic Documents, 45th Ed. Geneva: World Health Organization; 2006. Available from: http://www.who.int/governance/ eb/who_constitution_en.pdf. Accessed January 24, 2013.

29. Saracci R. The World Health Organization needs to reconsider its definition of health. BMJ. 1997;314(7091):1409-1410.

30. Byock I. Completing the continuum of cancer care: integrating life-prolongation and palliation. CA Cancer J Clin. 2000;50(2):123-132.

31. Twombly R. What's in a name: who is a cancer survivor? J Natl Cancer Inst. 2004;96(19):1414-1415.

32. Mehanna HM, Morton RP. Deterioration in quality-of-life of late (10-year) survivors of head and neck cancer. Clin Otolaryngol. 2006; 31(3):204-211.

33. Ganz PA. Monitoring the physical health of cancer survivors: a survivorship-focused medical history. J Clin Oncol. 2006;24(32): 5105-5111.

34. Stein KD, Syrjala KL, Andrykowski MA. Physical and psychological long-term and late effects of cancer. Cancer. 2008;112(Suppl 11): 2577-2592.

35. Harrington JM, Jones EG, Badger T. Body image perceptions in men with prostate cancer. Oncol Nurs Forum. 2009;36(2):167-172.

36. Yabroff KR, Lawrence WF, Clauser S, Davis WW, Brown ML. Burden of illness in cancer survivors: findings from a population-based national sample. J Natl Cancer Inst. 2004;96(17):1322-1330.

37. Ness KK, Mertens AC, Hudson MM, et al. Limitations on physical performance and daily activities among long-term survivors of childhood cancer. Ann Intern Med. 2005;143(9):639-647.

38. Deimling GT, Sterns S, Bowman KF, Kahana B. Functioning and activity participation restrictions among older adult, long-term cancer survivors. Cancer Investig. 2007;25(2):106-116.

39. Satariano WA. Comorbidities and cancer. In: Hunter CP, Johnson KA, Muss HB, editors. Cancer in the Elderly. New York, NY: Marcel Dekker; 2000:477-499. 
40. Piccirillo JF, Tierney RM, Costas I, Grove L, Spitznagel EL Jr. Prognostic importance of comorbidity in a hospital-based cancer registry. JAMA. 2004;291(20):2441-2447.

41. Asmis TR, Ding K, Seymour L, et al. Age and comorbidity as independent prognostic factors in the treatment of non small-cell lung cancer: a review of National Cancer Institute of Canada Clinical Trials Group trials. J Clin Oncol. 2008;26(1):54-59.

42. Hudson MM. A model for care across the cancer continuum. Cancer. 2005;104(Suppl 11):2638-2642.

43. Tyrer J, Duffy SW, Cuzick J. A breast cancer prediction model incorporating familial and personal risk factors. Stat Med. 2004;23(7): $1111-1130$.

44. Pai SI, Westra WH. Molecular pathology of head and neck cancer: implications for diagnosis, prognosis, and treatment. Annu Rev Pathol. 2009;4:49-70.

45. Hegelson VS, Snyder P, Seltman H. Psychological and physical adjustment to breast cancer over 4 years: identifying distinct trajectories of change. Health Psychol. 2004;23(1):3-15.

46. Kershaw T, Northouse L, Kritpracha C, Schafenacker A, Mood D. Coping strategies and quality of life in women with advanced breast cancer and their family caregivers. Psychol Health. 2004;19(2):139-155.

47. Tarakeshwar N, Vanderwerker LC, Paulk E, Pearce MJ, Kasl SV, Prigerson HG. Religious coping is associated with the quality of life of patients with advanced cancer. J Palliat Med. 2006;9(3):646-657.

48. Berry DL, Ellis WJ, Woods NF, Schwien C, Mullen KH, Yang C. Treatment decision-making by men with localized prostate cancer: the influence of personal factors. Urol Oncol. 2003;21(2):93-100.

49. Conti-Becker A. Between the ideal and the real: reconsidering the International Classification of Functioning, Disability and Health. Disabil Rehabil. 2009;31(25):2125-2129.

50. Renehan AG, Tyson M, Egger M, Heller RF, Zwahlen M. Body-mass index and incidence of cancer: a systematic review and meta-analysis of prospective observational studies. Lancet. 2008;371(9612): $569-578$.

51. Bradley CJ, Givens CW, Roberts C. Race, socioeconomic status, and breast cancer treatment and survival. J Natl Cancer Inst. 2002;94(7):490-496.

52. Molina JR, Yang P, Cassivi SD, Schild SE, Adjei AA. Non-small cell lung cancer: epidemiology, risk factors, treatment and survivorship. Mayo Clin Proc. 2008;83(5):584-594.

53. Schwartz AG, Wenzlaff AS, Prysak GM, et al. Reproductive factors, hormone use, estrogen receptor expression and risk of non small-cell lung cancer in women. J Clin Oncol. 2007;25(36):5785-5792.

54. Howe TJ. The ICF contextual factors related to speech-language pathology. Int J Speech Lang Pathol. 2008;10(1-2):27-37.

55. Stewart M. Towards a global definition of patient centred care. $B M J$. 2001;322(7284):344-345.

56. Geyh S, Peter C, Muller R, et al. The personal factors of the International Classification of Functioning, Disability and Health in the literature - a systematic review and content analysis. Disabil Rehabil. 2011;33(13-14):1089-1102.

57. Mead N, Bower P. Patient-centredness: a conceptual framework and review of the empirical literature. Soc Sci Med. 2000;51(7): $1087-1110$.
58. World Health Organization. Health Promotion Glossary. Geneva: World Health Organization; 1998. Available from: http://www.who. int/healthpromotion/about/HPR\%20Glossary\%201998.pdf. Accessed January 24, 2013.

59. Cieza A, Stucki G. The International Classification of Functioning Disability and Health: its development process and content validity. Eur J Phys Rehabil Med. 2008;44(3):303-313.

60. Geyh S, Cieza A, Kollertis B, Grimby G, Stucki G. Content comparison of health-related quality of life measures used in stroke based on the International Classification of Functioning, Disability And Health (ICF): a systematic review. Qual Life Res. 2007;16(5):833-851.

61. Cruice M. The contribution and impact of the International Classification of Functioning, Disability and Health on quality of life in communication disorders. Int J Speech Lang Pathol. 2008;10(1-2):38-49.

62. Frick E, Borasio GD, Zehentner H, Fischer N, Bumeder I. Individual quality of life of patients undergoing autologous peripheral blood stem cell transplantation. Psychooncology. 2004;13(2):116-124.

63. Moons P, Marquet K, Budts W, De Geest S. Validity, reliability and responsiveness of the "Schedule for the Evaluation of Individual Quality of Life - Direct Weighting" (SEIQoL-DW) in congenital heart disease. Health Qual Life Outcomes. 2004;2:27-35.

64. Llewellyn CD, McGurk M, Weinman J. Are psycho-social and behavioural factors related to health related-quality of life in patients with head and neck cancer? A systematic review. Oral Oncol. 2005;41(5):440-454.

65. Jansen L, Koch L, Brenner H, Arndt V. Quality of life among long-term ( $\geq 5$ years) colorectal cancer survivors - systematic review. Eur $J$ Cancer. 2010;46(16):2879-2888.

66. Chida Y, Hamer M, Wardle J, Steptoe A. Do stress-related psychosocial factors contribute to cancer incidence and survival? Nat Clin Pract Oncol. 2008;5(8):466-475.

67. Mehanna HM, de Boer MF, Morton RP. The association of psycho-social factors and survival in head and neck cancer. Clin Otolaryngol. 2008;33(2):83-89.

68. Cancer: fact sheet no 297 [webpage on the Internet]. Geneva: World Health Organization; 2012. Available from: http://www.who.int/ mediacentre/factsheets/fs297/en/. Accessed November 1, 2012.

69. McCabe C, Begley C, Collier S, McCann S. Methodological issues related to assessing and measuring quality of life in patients with cancer: implications for patient care. Eur J Cancer Care (Engl). 2008;17(1):56-64.

70. Bottomley A. The cancer patient and quality of life. Oncologist. 2002;7(2):120-125.

71. McDougall J, Wright V, Rosenbaum P. The ICF model of functioning and disability: incorporating quality of life and human development. Dev Neurorehabil. 2010;13(3):204-211.

72. Ueda S, Okawa Y. The subjective dimension of functioning and disability: what is it and what is it for? Disabil Rehabil. 2003;25(11-12): 596-601.

73. Ravenek MJ, Skarakis-Doyle E, Spaulding SJ, Jenkins ME, Doyle PC. Enhancing the conceptual clarity and utility of the International Classification of Functioning, Disability and Health: the potential of a new graphic representation. Disabil Rehabil. October 17, 2012. [Epub ahead of print.]

\section{Publish your work in this journal}

The Journal of Multidisciplinary Healthcare is an international, peerreviewed open-access journal that aims to represent and publish research in healthcare areas delivered by practitioners of different disciplines. This includes studies and reviews conducted by multidisciplinary teams as well as research which evaluates the results or conduct of such teams or

\section{Dovepress}

healthcare processes in general. The journal covers a wide range of areas and welcomes submission from practitioners at all levels, from all over the world. The manuscript management system is completely online and includes a very quick and fair peer-review system. Visit http://www.dovepress.com/testimonials.php to read real quotes from published authors. 